\section{P14 PHYSICAL ACTIVITY INTERVENTIONS FOR OVERWEIGHT AND OBESITY DURING PREGNANCY: A SYSTEMATIC REVIEW OF THE CONTENT OF BEHAVIOUR CHANGE INTERVENTIONS}

${ }^{1} \mathrm{C}$ Flannery ${ }^{*},{ }^{2} \mathrm{M}$ Fredrix, ${ }^{3}$ EK Olander, ${ }^{4} \mathrm{FM}$ McAuliffe, ${ }^{2} \mathrm{M}$ Byrne, ${ }^{1} \mathrm{PM}$ Kearney. ${ }^{1}$ School of Public Health, University College Cork, Cork, Ireland; ${ }^{2}$ School of Psychology, National University of Ireland, Galway, Galway, Ireland; ${ }^{3}$ Centre for Maternal and Child Health Research, School of Health Sciences, City, University of London, London, UK; ${ }^{4}$ Perinatal Research Centre, School of Medicine, University College Dublin, National Maternity Hospital, Dublin, Ireland

\subsection{6/jech-2019-SSMabstracts. 165}

Aim The aim of this study was to identify and summarise the effectiveness of physical activity (PA) interventions on PA levels for pregnant women with overweight and obesity, with a specific emphasis on the behaviour change techniques (BCTs) employed.

Methods A systematic review and meta-analysis of PA intervention studies using the PRISMA statement was conducted. Searches were conducted of eight databases in January 2018. Strict inclusion/exclusion criteria were employed. Primary outcome measures included change in PA levels, subjectively or objectively measured with physical fitness as a secondary outcome. The BCT taxonomy V1 was used to identify BCTs. Meta-analyses using random effect models assessed the intervention effects on PA. Other PA outcomes were summarised in a narrative synthesis.

Results From 8024 studies, 17 met the inclusion criteria. Of these, 11 studies provided data suitable for inclusion in a meta-analysis. Significant increases were found for metabolic equivalent (SMD $0.38 \quad[0.07,0.70], Z=2.40 \quad \mathrm{P}=0.02$ ) and physical fitness $\left(\mathrm{VO}_{2} \max \right)(\mathrm{SMD} 0.55$ [0.34, 0.75], $\mathrm{Z}=5.20$ $\mathrm{P}=<0.001$ ). Six additional studies were narratively described, five of which reported an increase in PA for the intervention group versus the control. 'Self-monitoring of behaviour' was the most frequently used BCTs (70.6\%), with 'social support' unique to this population.

Discussion This review revealed a slight increase in PA for pregnant women with overweight and obesity. However, these conclusions are tentative because of the poor methodological quality of the included studies. A range of BCTs clusters that could be used to help improve physical activity levels during pregnancy was identified, including: 'goals and planning', 'feedback and monitoring' and 'shaping knowledge' with 'social support' being unique to this population. Future studies should consider PA measures carefully so that studies can be meaningfully compared and intervention developers need to use recognised and standardised taxonomies so that BCTs can be accurately assessed.

\section{P15 INTERPREGNANCY WEIGHT CHANGE AND PREMATURE BIRTH: FINDINGS FROM A POPULATION-BASED COHORT IN THE SOUTH OF ENGLAND}

${ }^{1} \mathrm{G}$ Grove, ${ }^{1} \mathrm{~N}$ Ziauddeen*, 1,2NA Alwan. ${ }^{1}$ School of Primary Care and Population Sciences, Faculty of Medicine, University of Southampton, Southampton, UK; ${ }^{2}$ NIHR Southampton Biomedical Research Centre, University of Southampton and University Hospital Southampton NHS FT, Southampton, UK

10.1136/jech-2019-SSMabstracts. 166

Background Premature birth $(<37$ weeks gestation) is linked to maternal weight. However, the impact of maternal interpregnancy weight change on premature birth is unclear. The objective of this study was to examine the association between maternal interpregnancy weight change and premature birth, in a population-based cohort.

Methods Data from 2003 to 2018 from one maternity centre in England was used to produce two cohorts. The primary cohort ( $n=14,961$ women) consisted of first and second livebirth pregnancies. The secondary cohort ( $n=5,108$ women) consisted of second and third live-birth pregnancies. Logistic regression was used to look for associations between interpregnancy weight change and premature birth, whilst adjusting for covariates at second or third pregnancy (maternal age, ethnicity, educational achievement, employment status, co-morbidities, pregnancy complications, smoking status, previous premature birth, sex of the infant, fertility treatment), BMI and gestational age at initial pregnancy booking appointment and interpregnancy interval. Subgroup analyses were performed by booking BMI group at initial pregnancy.

Results More women gained weight $\left(>1 \mathrm{~kg} / \mathrm{m}^{2}\right.$, primary cohort: $47.1 \%$, secondary cohort: $46.5 \%$ ) than lost weight (>1 kg/m², primary cohort: $15.8 \%$, secondary cohort: $18.1 \%$ ) between pregnancies. The median weight change was $0.8 \mathrm{~kg} /$ $\mathrm{m}^{2}$ between pregnancies in both primary (IQR -0.4 to $2.4 \mathrm{~kg}$ $\mathrm{m}^{2}$ ) and secondary (IQR -0.5 to $2.4 \mathrm{~kg} / \mathrm{m}^{2}$ ) cohorts.

Primary cohort $3.4 \%(\mathrm{n}=514)$ of second pregnancy births were premature births. Weight loss $\left(>3 \mathrm{~kg} / \mathrm{m}^{2}\right)$ was associated with increased risk of premature birth (adjusted odds ratio (aOR) 3.50 , 95\% Confidence Interval (CI) 1.78-6.88), in women who were normal weight (BMI $18.5-25 \mathrm{~kg} / \mathrm{m}^{2}$ ) at the start of their first pregnancy. Weight gain $>1 \mathrm{~kg} / \mathrm{m}^{2}$ was not associated with the outcome in any of the initial BMI categories.

Secondary cohort $4.2 \% \quad(n=212)$ of third pregnancy births were premature births. Losing $>3 \mathrm{~kg} / \mathrm{m}^{2}$ was associated with increased risk of premature birth (aOR 2.01, 95\% CI 1.053.87) when analysing the whole sample, but not when restricting the analysis to overweight or obese women at the start of the second pregnancy.

Conclusion Interpregnancy weight loss in women who were normal weight in their first pregnancy was associated with increased risk of premature birth. No such association was found when the analysis was restricted to women who were overweight or obese in their previous pregnancy whether it was first or second. Interpregnancy weight gain was not associated with premature birth. Current national guidelines encouraging obese women to lose weight before a pregnancy should be followed, in order to reduce other maternal and offspring adverse outcomes of maternal obesity.

\section{P16 AN INTERDISCIPLINARY STUDY OF HISTORICAL AND EPIDEMIOLOGICAL TRANSITIONS IN URBAN CARIBBEAN FOODSCAPES: UNDERSTANDING THE PAST TO ENHANCE FUTURE NUTRITION STRATEGIES}

${ }^{1} \mathrm{C}$ Guell ${ }^{*},{ }^{2} \mathrm{H}$ Altink, ${ }^{3} \mathrm{M}$ Moore, ${ }^{4} \mathrm{M}$ McMorris, ${ }^{1} \mathrm{~K}$ Morrissey, ${ }^{4} \mathrm{M}$ Smith, ${ }^{5} \mathrm{~N}$ Unwin, ${ }^{3} \mathrm{~A}$ Williams, ${ }^{3}$ I Govia. ${ }^{1}$ European Centre for Environment and Human Health, University of Exeter, Truro, UK; ${ }^{2}$ Department of History, University of York, York, UK; ${ }^{3}$ Caribbean Institute for Health Research, University of the West Indies, Kingston, Jamaica; ${ }^{4}$ Department of History and Archaeology, University of the West Indies, Kingston, Jamaica; ${ }^{5} \mathrm{MRC}$ Epidemiology Unit, University of Cambridge, Cambridge, UK

10.1136/jech-2019-SSMabstracts. 167

Background Healthy environments need to be created that enable easy access to healthy and nutritious food. Complex 
and expanding urbanisation has had deleterious effects on the diet and health of city dwellers; yet urban planning offers a potential mechanism for public health strategies. To inform such policy efforts in the Caribbean, we conducted a cross-disciplinary study to understand historical and epidemiological transformations in Kingston, Jamaica. With this case study, we aimed to develop methods and conceptual tools for investigating the historical social, political and economic contexts that have shaped contemporary foodscapes and can inform future nutrition strategies.

Methods Caribbean historians analysed principally online Jamaican newspaper archives to examine changes in food availability, affordability and consumption, and public discourses around eating, particularly fast food and other types of unhealthy food, from 1945 to present. Public health researchers undertook a scoping review to map available regional health survey databases for local nutrition and related health data, to examine major epidemiological trends in nutrition for the same time frame and assess its impact on noncommunicable diseases. Geographical information scientists mapped the health and historical data to produce an interactive map of the evolution of Kingston's foodscape.

Results

Preliminary historical findings include the increasing popularisation of commercial, more quickly prepared meals between the 1950s and 1970s; a national campaign to promote locally produced food crops in the diet to address food scarcity in the late 1970s; and the proliferation of United States fast food chains in Kingston in the 1980s. The health data review found thirteen relevant health or nutrition surveys on malnutrition including childhood stunting rates, nutrient deficiency and obesity rates. Few predate the 1980s and many only offer national (versus city level) data. Annual health and demographic reports pre- and post-independence in 1962 might be able to fill data gaps. For the geographical information systems map, we had to identify relevant historical address points, as well as identify and digitalise historical maps from 1945 onwards onto which data can be layered.

Conclusion Structural approaches to public health require us to expand the scope of transdisciplinary research and build the methodological capacity to make 'non-traditional' connections. In this case study of the health history of a major Caribbean urban space, while not claiming causal relationships between historic and health trends, we suggest the important role that natural events, international and local policies, and advertising driven economies play in the production of 'unhealthy' foodscapes over time.

This project was funded by the UK Medical Research Council - Arts and Humanities Research Council GCRF Global Public Health Partnership Award [Grant number: MR/ R024324/1].

\section{\begin{tabular}{|l|l}
\hline P17 DIETARY INFLAMMATORY POTENTIAL AND RISK OF \\
\hline
\end{tabular} BREAST CANCER: RESULTS FROM A CASE-CONTROL STUDY IN FRANCE}

\footnotetext{
1,2M Hajji*, 1,2E Cordina-Duverger, 1,2P Guénel. 'Inserm U1018, Center for Research in Epidemiology and Population Health, Villejuif, France; ${ }^{2}$ Paris-Sud University, Paris Saclay, Villejuif, France
}

10.1136/jech-2019-SSMabstracts. 168

Background Diet is known as one of the major modifiable risk factors for the regulation of chronic inflammation.
Epidemiologic studies that have examined the association between dietary inflammatory potential and breast cancer risk have been inconsistent. The aim of this study was to assess the association between the Adapted Dietary Inflammatory Index (ADII) and breast cancer risk.

Methods The study was conducted using data from the CECILE study, a population-based case-control study in 2 French departments (Ille-et-Vilaine and Côte d'Or). The group of cases included women aged 25-75 years with a cancer of the breast diagnosed between 2005 and 2007. Controls were selected at random from the telephone directories and were frequency-matched to the cases by age and department.

Information on socio-demographic characteristics, lifestylerelated factors, hormonal and reproductive history, previous medical conditions, and family history of breast cancer were obtained from a structured questionnaire during in-person interviews. Information on dietary habits the year prior to inclusion was self-reported by the study subjects using a 150 items food frequency questionnaire.

The ADII for each subject was calculated as the sum of the standardized energy-adjusted intake of each dietary component weighted by its dietary inflammatory score, as described by (van-Woudenbergh et al, Am J Clin Nutr, 2013).

Odds ratios (ORs) and 95\% confidence intervals (CIs) were calculated from multivariable logistic regression after controlling for potential confounders selected among breast cancer risk factors.

Results The analysis was based on 840 cases and 908 controls who completed the food frequency questionnaire. The OR for breast cancer in women in the highest quartile of ADII as compared to women in the lowest quartile, was 1.43 (95\% CI: 1.07-1.90) and there was a significant trend of increasing risk with increasing ADII. The corresponding OR in menopausal women was $1.58,95 \% \mathrm{CI}$ : $1.08-2.31$, while no statistically significant association was observed in premenopausal women

Conclusion Our results suggest that a pro-inflammatory diet increases the risk of developing breast cancer among postmenopausal women.

\section{P18 MULTILEVEL LATENT CLASS MODELLING OF SIMULATED HEALTHCARE PROVIDER-LEVEL CAUSAL EFFECTS IN OBSERVATIONAL DATA}

1,2WJ Harrison*, ${ }^{2} \mathrm{PD}$ Baxter, 1,2,3 MS Gilthorpe. 'Leeds Institute for Data Analytics, University of Leeds, Leeds, UK; ${ }^{2}$ School of Medicine, University of Leeds, Leeds, UK; ${ }^{3}$ The Alan Turing Institute, London, UK

\subsection{6/jech-2019-SSMabstracts. 169}

Background Healthcare provider performance is commonly assessed using patient outcomes, e.g. survival rates. Patient characteristics that may affect outcomes in the absence of genuine provider-level differences must therefore be balanced across providers to ensure a fair comparison. There are many methods that can accommodate this patient 'casemix' but none that also allow the assessment of provider-level covariate effects, i.e. the potential causes of performance differences. We aim to demonstrate the utility of multilevel latent class (MLC) modelling to identify causal provider-level covariate effects after accommodating patient differences.

Methods We simulated data for patients and providers, based on a previously utilised real-world dataset of patients diagnosed with colorectal cancer. Age at diagnosis, sex and 